\title{
Design of driving mechanism of racing ship model
}

\author{
Yuanyuan Tang, Jie Chen, Yehui Chen, Zixuan Tang
}

Nantong Institute of Technology

Author: Yuanyuan Tang, November 15, 1988,female, Han, Suqian Jiangsu, Undergraduate, Nantong Institute of Technology, 226002, Research direction: Ship design

Corresponding author: Jie Chen, January 21,1990,female, Han, Xuzhou Jiangsu, Bachelor degree, Nantong Institute of Technology, 226002, Research direction: Ship design

Preface: Model is the ship model, which is strictly scaled down according to the shape, structure, color and even interior parts of the real ship ${ }^{[1]}$.In the environment with poor water quality, the existing model driving structure is easy to make the propeller entangled with debris, which affects the driving mechanism of the model, the speed of the model, and even the movement of the model.

Fund projects: Jiangsu students' innovation and Entrepreneurship training project;

Project Name: Study on the speedability of racing ship;

Project Number :201912056012Y

Abstract: This paper introduces a design of a racing boat model driving mechanism. By improving and optimizing the structure of the ship model driving mechanism, the propeller of the ship model driving mechanism is protected, so as to avoid the problems of the propeller's rotation and the ship model's movement which are affected by the debris wound on the propeller during rotation. The sealing effect of the ship model driving mechanism is better than that of the existing ship model driving mechanism.

Keywords: Ship model; Driving mechanism; speed

\section{Design scheme}

In order to solve the above problems, the author designed a model driving mechanism, installed on the ship model ontology, model ontology runs at the bottom of shaft hole, inside the shaft hole is sealed with collar, sleeve through the clamp fixed in the above model ontology, axle sleeve head fixed bridge connection, connection seat of fixed has described with collar coaxial motor, motor output shaft through the shaft coupling and install it inside the shaft sleeve shaft, shaft and shaft sleeve with sealing ring, between the tail end of the rotating shaft extends to the outside and connected to the propeller model ontology. The driving mechanism of the ship model is simple and compact, convenient and quick to install, with good coaxiality, stable transmission and good waterproof effect.

\section{Design of ship model driving mechanism}

The driving mechanism of the ship model (as shown in Figure 1) includes the ship model 1. The installation platform 13 is located near the bottom inside the ship model 1, and the installation platform 13 is provided with motor 3. The output end of motor 3 is provided with a rotating shaft 32. The rotor shaft 32 passes through the tail end of model 1 , and one end of the rotor shaft 32 protruding out of model 1 is provided with a propeller 4 , and the tail end of model 1 is aligned with the position of propeller 4 , and a baffle 12 is provided with a filter plate 2 at the rear end of

Copyright (C) 2020 Yuanyuan Tang et al.

doi: 10.18282/le.v9i5.1192

This is an open-access article distributed under the terms of the Creative Commons Attribution Non-Commercial License

(http://creativecommons.org/licenses/by-nc/4.0/), which permits unrestricted non-commercial use, distribution, and reproduction in any medium, provided the original work is properly cited. 
baffle 12; Installation platform 13 facilitates the installation of motor 3, which facilitates the installation of the driving components. It facilitates the driving components to drive ship model 1. Motor 3 facilitates the rotation of propeller 4 , which facilitates the movement of ship model 1 through propeller 4 rotation. The shaft 32 is convenient to connect the output end of motor 3 to propeller 4 , thus facilitating the rotation of propeller 4 and driving the movement of model 1. Baffle 12 is convenient for shielding the side of propeller 4, so as to avoid entangled sundries on propeller 4 . Filter plate 2 is convenient for shielding the back end of propeller 4, so as to protect propeller 4 and avoid entangling sundries on propeller 4 . This structure makes the model 1 drive structure of propeller 4 are protected, to avoid clutter in the propeller rotates 4 winding affect the rotation of the propeller 4 , will affect the problem of the model 1 mobile and this kind of model 1 drive structure compared with the existing model 1 drive better sealing effect, thus to avoid the problem of internal water model 1. Casing 11 is provided at the back end of ship model 1 at the alignment position of rotating shaft 32, and sealing bearing 111 is provided at the back end of casing 11 . The casing 11 is convenient to be fitted on the shaft 32 , so as to protect the shaft 32 and avoid the corrosion problem of the shaft 32 easily, thus increasing the service life of the shaft 32. The sealed bearing 111 is convenient for the rear end of casing 11 to be sealed to avoid water from the rear end of casing 11 to enter the interior of ship model 1. Meanwhile, the sealed bearing 111 is also convenient for the use of rotating shaft 32 , so as to facilitate the rotation of rotating shaft 32 . The rear end of the baffle 12 and the ship model 1 are fixed together, and the inner ring of the baffle 12 is set with a limit table 121 near the rear end.This structure is convenient for the baffle 12 to protect the propeller 4 stably, while the limit table 121 is convenient for the installation of the filter plate 2, which facilitates the installation and disassembly of the filter plate 2, and also avoids the assembly of the drive components.
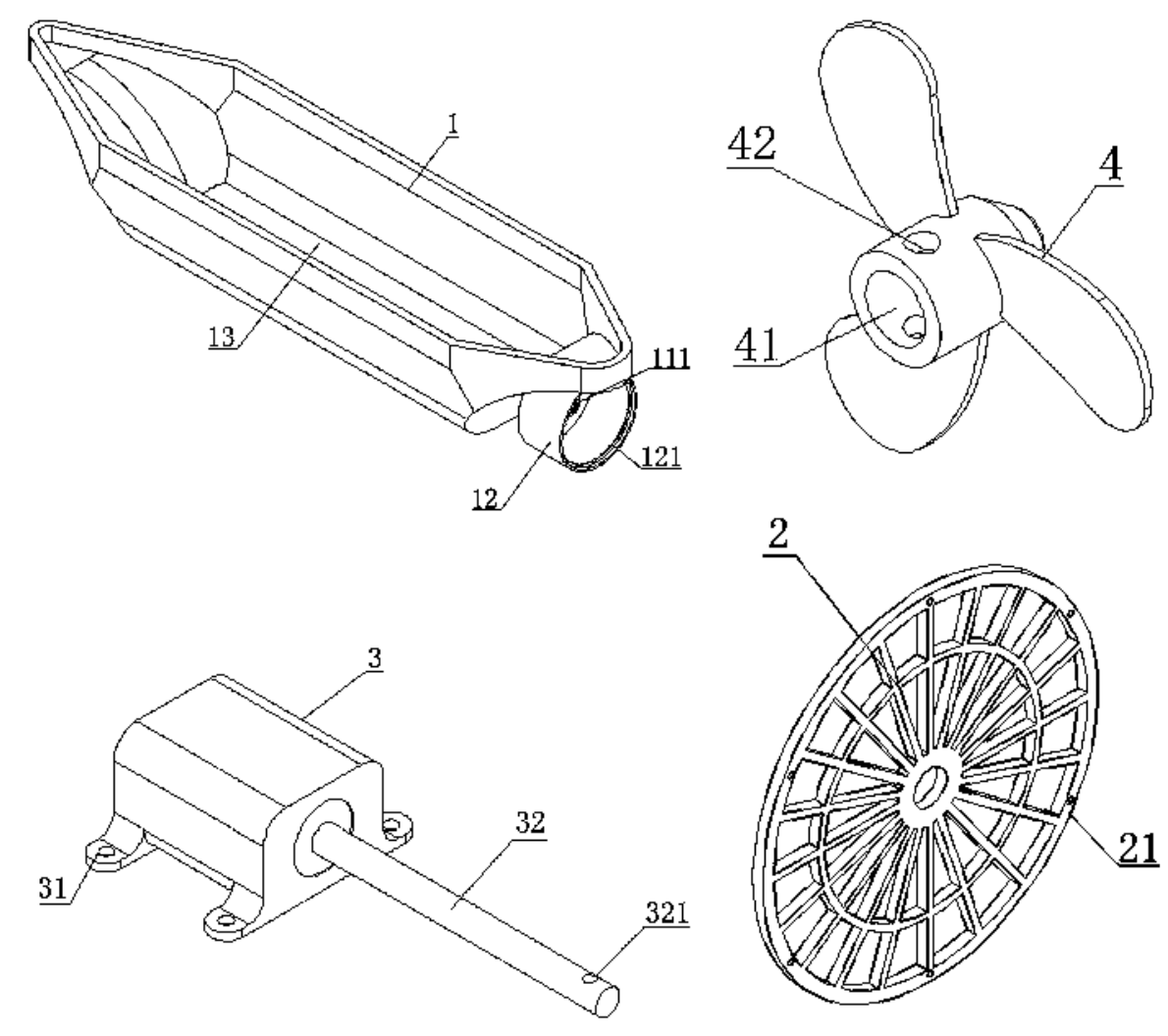

Figure 1 Driving mechanism of racing ship model

\section{Installation method}

When installation model 1 planking disassembly report first, then install the motor 3 and axis of 32 placed in Taiwan on 13 , then push the motor 3 , makes the rotating shaft 32 and out through the casing model 1, the axis of 32 and sealed bearing 111 interference connection, through the bolt will be motor fixed on the mounting plate 13 and 3 for the motor with 3 power, then 4 sets of propeller and propeller by bolt in the end of the axis of 324 and axis of 32 together, finally 
will filter plate 2 card on the limit at 121 and through the bolt will filter plate on the limit at 121 with 2 can be used.

\section{Conclusion}

It has been proved by practice that by improving and optimizing the structure of the ship model driving mechanism, the propeller of the ship model driving structure can be protected, so as to avoid the influence of the propeller rotation by winding up with debris during the propeller rotation, which provides guarantee for the moving operation of the ship model.In addition, the model drive structure has a better sealing effect than the existing model drive mechanism, thus solving the problem of water inlet in the model.

\section{Reference}

1. Lu Tiane, Fang Shun. Research on the Influence of Jiangsu Folk Culture on the design and structure of ancient Ships [J]. Popular Literature and Art, 2020(09):60-61.

2. Zhang Weiming, ZHANG Yuangang. Research on the Development of Marine Power Plant [J]. Internal Combustion Engine \& Parts,2017(17):39-40. 